\title{
Channel equalization based on data reuse LMS algorithm for shallow water acoustic communication
}

Feng TONG

\author{
Bridget Benson Ying $\mathrm{Li}^{2} \quad$ Ryan Kastner
}

\begin{abstract}
In recent years, there has been increasing interest in the design of underwater acoustic modems for marine environmental monitoring, underwater structure inspection and sea bottom resource exploitation. As underwater acoustic channels pose difficulties such as multipath, time-space selectivity, frequency dependent noise, and Doppler shifts on transmission, research on adaptive equalizers play an important role in the design of underwater modems. This paper presents a data reuse least mean square (DR-LMS) algorithm to achieve equalization performance with low computational complexity to facilitate a practical hardware implementation. Experimental results obtained in physical shallow water channels demonstrate the effectiveness of the proposed method compared to classic LMS (Least mean square) and RLS (Recursive least square) algorithms.
\end{abstract}

\section{I . INTRODUCTION}

There has been an increasing interest in underwater communications for many marine applications such as environmental (pollution, coral reef, seismic, ocean current, etc.) monitoring, underwater structure inspection (oil platform, pipeline, undersea tunnel, etc.), oceanographic investigation, and sea bottom resource exploitation. However, due to the difficulties encountered in underwater acoustic (UWA) channels, digital communications through UWA channels are much more difficult than those in other media, such as the radio channel ${ }^{[1]}$. One of the main obstacles for reliable high speed UWA communications through severely band-limited UWA channels is intersymbol interference (ISI) ${ }^{[2-5]}$. While typical multipath spreads in the mobile radio channel are two or three symbol intervals, they increase to several tens of symbol intervals for moderate to high data rates in the shallow-water acoustic channel. Channel equalization provides an effective solution to overcome ISI.

There is a great amount of literature on adaptive channel equalization algorithms (especially on the classic LMS and RLS algorithms), but there is a lack of literature on their adaptability to the UWA channel. The UWA time variant channel requires that the equalization algorithm provide fast convergence to ensure high tracking performance. However, the lack of suitable power resources in many UWA application scenarios requires the algorithm to have low-complexity to allow for a power efficient implementation. Some efforts have been made to derive adaptive algorithms that converge faster or are more efficient from a complexity point of view than the classical LMS algorithm as well as the RLS algorithm ${ }^{[6-9]}$, but few efforts have addressed both fast convergence and low complexity for use in the underwater channel.

In this paper, we present DR-LMS as a channel equalization algorithm capable of achieving both the algorithm performance and low computation complexity required for practical use in the underwater acoustic channel. DR-LMS is a fast-converging algorithm that avoids any division operation making it an attractive algorithm for an efficient hardware design. Though the DR LMS algorithm has been theoretically investigated in several papers ${ }^{[6-8]}$, there are few works that reported its performance in an experimental setting. Thus, we also report experimental results showing the algorithm's performance on a real UWA communication link.

Besides the DR-LMS algorithm, we also adopt DFE (Decision feedback equalizer) and FSE structures (fractionally space equalizer, FSE) to guarantee performance under hostile shallow water channels ${ }^{[1]}$. Finally, we compare DR-LMS with classic LMS and RLS will to verify its performance improvement.

The rest of the paper is organized as follows: Section II presents the basis of the classic and DR LMS algorithms. Section III introduces the system design and sea experiment configuration. The Section IV provides the experimental results. We conclude in section $\mathrm{V}$.

\section{II . BASIS OF DR-LMS}

Firstly, let us define the classic LMS algorithm. Its iteration formula of coefficients $W_{k}$ updating is ${ }^{[9]}$ : 
$X_{k}=\left[x_{k}, x_{k+1}, \ldots, x_{k+L-1}\right] \quad k=1,2, \ldots N$

$e_{k}=d_{k}-X_{k}^{T} W_{k}$

$W_{k+1}=W_{k}+2 \mu \cdot e_{k} \cdot X_{k}$

Where $u$ is a gain constant used to control the convergence rate, $e_{k}$ is the error of an adaptive system, $N$ is the length of the input and reference signal, $L$ is the length of the filter coefficients, $d_{k}$ and $x_{k}$ are the reference signal and input signal respectively. The original tap weights, $W_{0}$, are generally set to zero.

The first data reuse adaptive algorithm was introduced by Shaffer and Williams ${ }^{[7]}$ for the LMS algorithm and consists of reusing the same data $\mathrm{N}$ times. The data reuse LMS (DR-LMS) algorithm is then given by the following equations:

Step1: Initialization $: i=0$

$e_{k}=e_{k, 0}, W_{k, 0}=W_{k}$,

Step 2: Loop: While $\quad i \leq N-1$

$e_{k, i}=d_{k}-X_{k}^{T} W_{k, i}$

$W_{k, i+1}=W_{k, i}+2 \mu \cdot e_{k, i} \cdot X_{k}, \quad i=i+1$

Step3: Update

$W_{k+1}=W_{k, N}, k=k+1 ; \quad$ Goto $\quad$ Step 1

Obviously, $N=1$ reduces to the classic LMS update. It also can easily be obtained that the weight updating equation can be further rewritten as:

$$
\begin{aligned}
& e_{k, i}=e_{k}\left[1-\mu X_{n}^{T} X_{n}\right]^{i}, i=0, \ldots, N-1 \\
& W_{k+1}=W_{k}+\frac{X_{n}}{X_{n}^{T} X_{n}} e_{k}\left\{1-\left[1-2 \mu X_{n}^{T} X_{n}\right]^{N}\right\}
\end{aligned}
$$

Thus when $N$, the DR-LMS is actually a special case of the normalized LMS (NLMS) algorithm which is defined as [6]:

$$
W_{k+1}=W_{k}+\frac{\alpha X_{n}}{X_{n}^{T} X_{n}} e_{k}
$$

Where $\alpha$ is a normalized step size. Therefore, theoretically it can be concluded that the DR-LMS algorithm will converge at the rate between that of LMS and NLMS. Compared to NLMS, DR-LMS does not need any division calculation thus simplifying the algorithm design for a practical hardware implementation.

\section{SYSTEM AND EXPERIMENT DESIGN}

The coherent UWA communication link used to test the algorithm performance is shown in Fig. 1. The link consists of two computers (one acting as a bit resource while the other acting as a signal recorder), a DA and AD card used for data output and acquisition, power amplifier, preamplifier, and two transducers (one for transmitting and the other for receiving).
The adaptive channel equalization algorithm described in the previous section was implemented in MATLAB and used for off-line processing of experimental data. In the DR-LMS algorithm implementation we used the small iteration numbers of $\mathrm{N}=2,3,4$ to reduce the complexity of a practical hardware design.

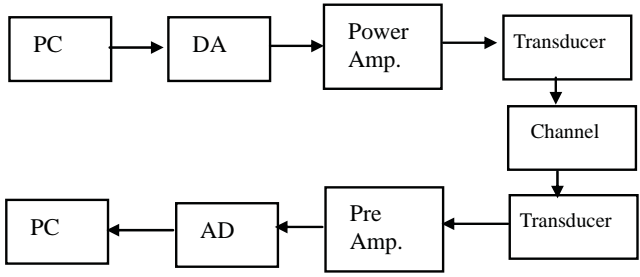

Fig .1 Bock chart of experimental UWA link

The signal frame consisted of an LFM (Linear frequency modulation) chirp to acquire synchronization and detect the channel, a guard time and the modulated data. The carrier frequency was $16 \mathrm{k} \mathrm{Hz}$, with the sampling rate at $96 \mathrm{ksps}$. The modulation format was QPSK, and the signals were transmitted at 6.4 kilobits per second. The bandwidth of transducer couple was $13-18 \mathrm{k} \mathrm{Hz}$.

The experiment in the ocean was carried out at Wuyuan Bay, Xiamen, China. The depth of the experiment area is approximately $7 \mathrm{~m}$ under the pier and $12 \mathrm{~m}$ offshore. The arrangement of the experiment is shown in Fig. 2. The transmit transducer was suspended from the pier at the depth of $5 \mathrm{~m}$ from the sea surface. Similarly, the receive transducer was suspended from a boat at the depth of $5 \mathrm{~m}$ from the sea surface. The distance between the transmitter and receiver was $2 \mathrm{~km}$.

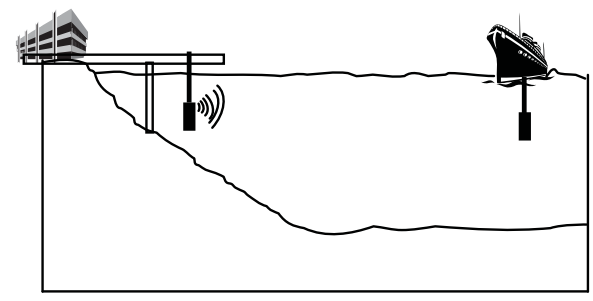

Fig.2 Sea experiment configuration

\section{EXPERIMENTAL RESULTS AND DISCUSSION}

The input to the equalizer is the raw received signal, brought to baseband using the nominal carrier frequency, sampled at $2 / \mathrm{T}$, and frame-synchronized using the LFM sync head. No phase synchronization or bit-timing adjustment is performed on the signal. The $T / 2$ spaced DFE structure equalizer contained a 32-tap feedforward filter and a 16-tap feedback filter.

For the purpose of comparison, classic LMS and RLS algorithm were also adopted to process the signal. With the forgotten factor of RLS algorithm set at 0.985 .

The channel response obtained with the LFM channel probe transmitted before the actual data is shown in Fig. 3. 
The channel probe can be used to determine roughly the extent as well as the pattern of the multipath. The channel response consists of the dominant component of arrivals whose delay spread is on the order of 15 symbol intervals. In addition, there is a distant cluster of weak arrivals, approximately 50 symbol intervals away.

The raw received signal recorded during the sea experiment has an SNR (Signal noise ratio) of $14 \mathrm{~dB}$. Shown in Fig.4 (a)(b)(c)(d)(e) are the equalization results of the LMS, RLS and the DR-LMS algorithm with iteration number $\mathrm{N}=2,3,4$ respectively. As seen by the output scatter plot, the performance of LMS and DR-LMS algorithms is of moderate quality compared to that of the RLS receiver (See Fig.4(b)). One may also observe that the classic LMS algorithm (See Fig.4(a)) as well as the DR-LMS with $\mathrm{N}=2$ (See Fig.4(c)) still contain some residual phase deviation caused by channel, which is adjusted to some extent in the DR-LMS receiver with $\mathrm{N}=3$ (See Fig.4(d))and N=4 (See Fig.4(e)). The performance comparison in the scatter plot output indicates that some performance improvement is achieved by DR-LMS algorithm with respect to the classic LMS.

The mean square error (MSE) results of the different algorithms are shown in Fig.5. It needs to be noted that here the MSE curve of DR-LMS receiver with iteration number $\mathrm{N}$ is directly obtained from the error at the $N$ th iteration. As we can observe from Fig.5, RLS yields better MSE than LMS, but DR-LMS with higher iteration number yields better MSE than RLS. However, for the DR-LMS receiver, a better MSE curve at the $N$ th iteration does not necessarily mean better equalization performance as the calculation of the equalizer tap depends on overall data reuse error, not simply on that of the final iteration, as indicated by equation [9].

The relationship between performance and the iteration number is verified at the estimated bit error rate (BER) output as shown in Tab. 1. We find that the DR-LMS receiver with $\mathrm{N}=2$ exhibits a superior BER result, $0.95 \%$, to the BER of classic LMS $(1.3 \%)$, and DR-LMS with $\mathrm{N}=3$ corresponds to an even better BER (0.92\%). But, further increasing the iteration number of DR-LMS does not result in further increasing the BER performance improvement, as the BER degrades from $0.92 \%$ to $1.15 \%$ when $\mathrm{N}$ increases from 3 to 4 . Thus, there is an optimal $\mathrm{N}$ value for DR-LMS receiver in view of the performance improvement introduced by data reuse iterations. In the shallow water acoustic communication scenario discussed here, $\mathrm{N}=3$ achieves the optimal result.

If we use the number of multiplications per iteration to determine the complexity of each algorithm for a filter with the number of taps $M$, the RLS receiver corresponds to the best BER performance $(0.4 \%)$ at the expense of a calculation burden increasing as the square of $\mathrm{M}^{[9]}$. On the other hand, the DR-LMS algorithm with $\mathrm{N}=3$ only requires approximately $2 \mathrm{NM}=6 \mathrm{M}$ multiplications to achieve only a slightly worse BER $(0.92 \%)$. When considering $\mathrm{M}=32$ for the feedforward filter and $\mathrm{M}=16$ for the feedback filter, the difference of algorithm complexity is quite high in this underwater scenario.

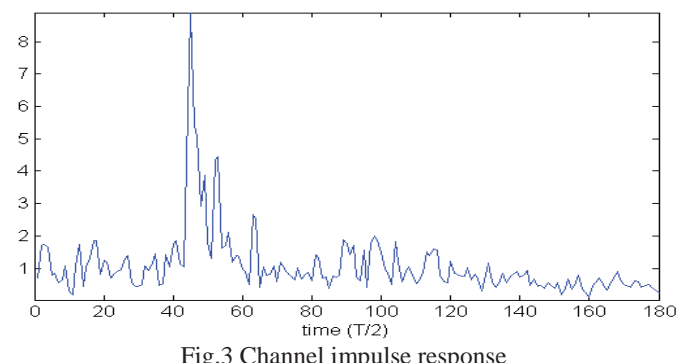

Fig.3 Channel impulse response

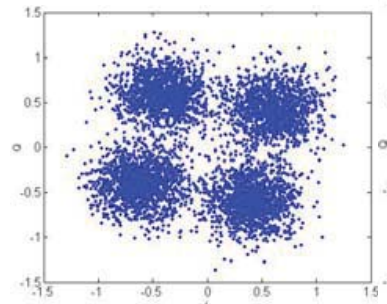

(a) LMS

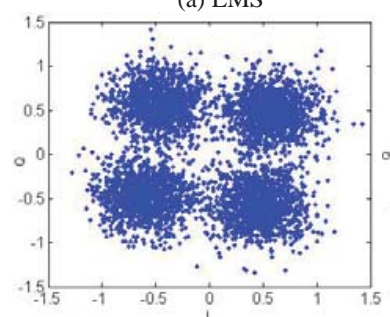

(c) DR-LMS N=2

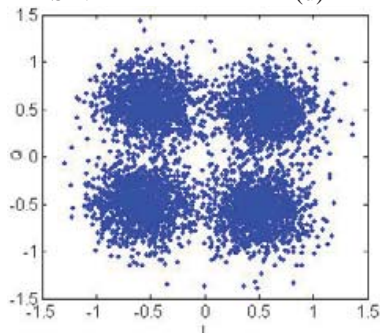

(e) DR-LMS N=4

Fig.4 Scatter plot of equalization result

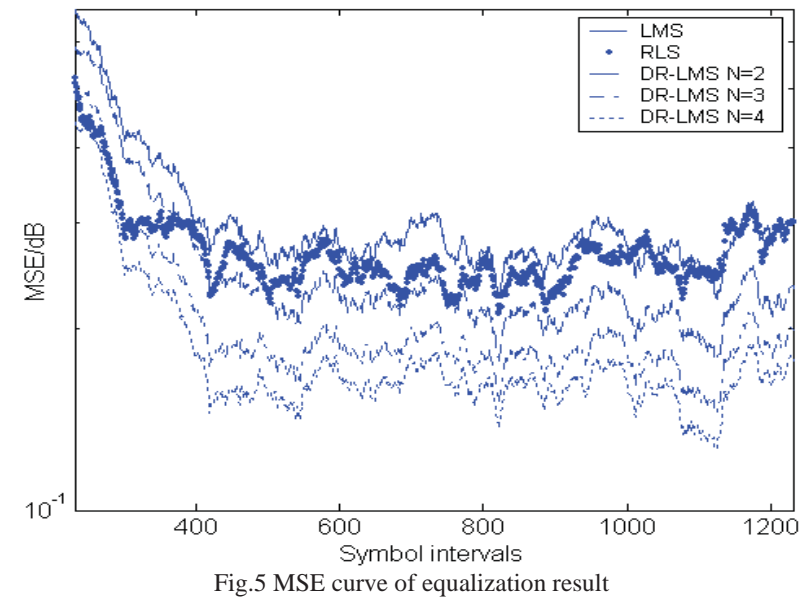


TABLE1. BER PERFORMANCE OF EQUALIZATION RESULT

\begin{tabular}{|c|c|}
\hline Equalization algorithm & Bit error rate (\%) \\
\hline LMS & 1.3 \\
\hline RLS & 0.4 \\
\hline DR-LMS, N=2 $\quad \mathrm{N}=3$ & 0.95 \\
\hline DR-LMS, $\quad \mathrm{N}=4$ & 0.92 \\
\hline DR-LMS, & 1.15 \\
\hline
\end{tabular}

\section{CONCLUSIONS}

In this paper, we have presented the theoretical foundation, system design and experimental result of the DR-LMS channel equalization technique to ensure reliable acoustic communications in a shallow water acoustic channel. In order to overcome the intersymbol interference caused by multipath propagation, the DR-LMS algorithm is incorporated with BSE-DFE structure to form an adaptive channel equalizer for the coherent acoustic communication link. The field test carried out in Wuyan Bay shows that the employment of DR-LMS can achieve some performance improvement against the classic LMS equalizer.

Previous theoretical analysis of DR-LMS algorithm has indicated that it will need an infinite data reuse iteration to attain the performance of NLMS ${ }^{[6]}$, making the algorithm less attractive for a practical implementation. However, the comparison result of our shallow water acoustic communication experiment show that a small iteration number is enough for DR-LMS to ensure some performance improvement over the classic LMS approach. Moreover, it is found that a higher iteration number will not always correspond to a better result, as the DR-LMS with $\mathrm{N}=3$ achieved the optimal BER output in our experiment. Furthermore, some enhancement in phase adjustment of DR-LMS algorithm can be noticed in our coherent acoustic communication data analysis.

With the increasing interest in dense, short-range and lost cost underwater networks used for marine environmental and structural applications ${ }^{[10,11]}$, attention must be paid to the design of simple, uncomplate, lost cost and energy-efficiency underwater acoustic modems. Thus, from a practical point of view, reliable and low-complexity algorithms such as DR-LMS have the potential of being developed and employed in this field.

\section{ACKNOWLEDGMENT}

The authors are grateful for the funding by a grant from Nature Science Foundation of China (NSFC) (No. 10704063) and China Scholarship Council (CSC) in support of the present research.

\section{REFERENCES}

[1] D.B.Kilfoyle, A. B. Baggeroer, "The State of art in underwater acoustic telemetry", IEEE J.Oceanic. Eng., Vol, 25(1), pp.4-24, 2000

[2] M.D.Green, J.A.Rice, "Channel-Tolerant FH-MFSK Acoustic Signaling for Undersea Communications and Networks", IEEE J.Oceanic. Eng., Vol, 25(1), pp.28-39, 2000
[3] M.Stojanovic, L.Freitag, "Acquisition of Direct Sequence Spread Spectrum Acoustic Communication Signals”, Oceans'2003 Proceedings, Vol. 1, pp.22-26, Sep. 2003

[4] J.G..Proakis, "Coded modulation for digital communication over rayleigh fading channels", IEEE.J..Oceanic. Eng., Vol, 21(8), pp.125-135, 1991

[5] E.M.Sozer, J.G..Proakis, M. Stojanovic, J.A.Rice, A.Benson, M.Hatch, "Direct sequence spread spectrum based modem for underwater acoustic communication and channel measurements", Oceans'99, MTS/IEEE, Riding the Crest into the $21^{\text {st }}$ Century, Vol.1,pp. 228-233. 1999

[6] Jacob Benesty, Tomas Gansler, "On data-reuse adaptive algorithms", International workshop on acoustic echo and noise control (IWAENC2003), Sept.2003, Kyoto, Japan

[7] S. Shaffer, C. S. Williams, "Comparison of LMS, alpha LMS, and data reusing LMS algorithms," Conference Record of the Seventeenth Asilomar Conference on Circuits,Systems and Computers, Nov. 1983, pp. 260-264.

[8] Tantikovit, S.Wang, M.Z. "An adaptive data-reuse interference cancellation scheme for DS-CDMA systems", 1997 International Conference on Information, Communications and Signal Processing, 1997. ICICS., 9-12 Sep 1997, pp: 854-857 vol.2, Singapore

[9] B. Farhang-Boroujeny, Adaptive filters-theory and applications, John Wiley \& Sons, Newyork, 1998

[10] Bridget Benson, Ali Irturk, Junguk Cho and Ryan Kastner, "Energy Benefits of Reconfigurable Hardware for Use in Underwater Sensor Nets", IEEE Reconfigurable Architectures Workshop, May 2009

[11] Ying Li, Bridget Benson, Ryan Kastner and Xing Zhang, "Bit Error Rate, Power and Area Analysis of Multiple Implementations of Underwater FSK", International Conference on Engineering of Reconfigurable Systems and Algorithms, July 2009 